\title{
oNAHES \\ BRCA2 mutation offers response and survival advantages
}

Women with BRCA1 or BRCA2 mutations have an increased risk of developing ovarian cancer. Therefore, as part of The Cancer Genome Atlas (TCGA) project, Wei Zhang and colleagues undertook a "comprehensive analysis of genomic, epigenetic, and clinical data in the TCGA database to identify not only clinically important associations with $B R C A 1$ and BRCA2 mutations but also gain genomic insight into the mechanisms," explains Zhang. The researchers evaluated the association between $B R C A 1$ and BRCA2 mutations and overall survival, progression-free survival, and chemotherapy response in 316 patients with high-grade serous ovarian cancer.

"The most significant finding was that mutations in BRCA2 but not in BRCA1 are associated with better progression-free and overall survival and better response to DNA-damaging platinum chemotherapy in patients with ovarian cancer," highlights Zhang. Using whole-exome deepsequencing Zhang's team also showed

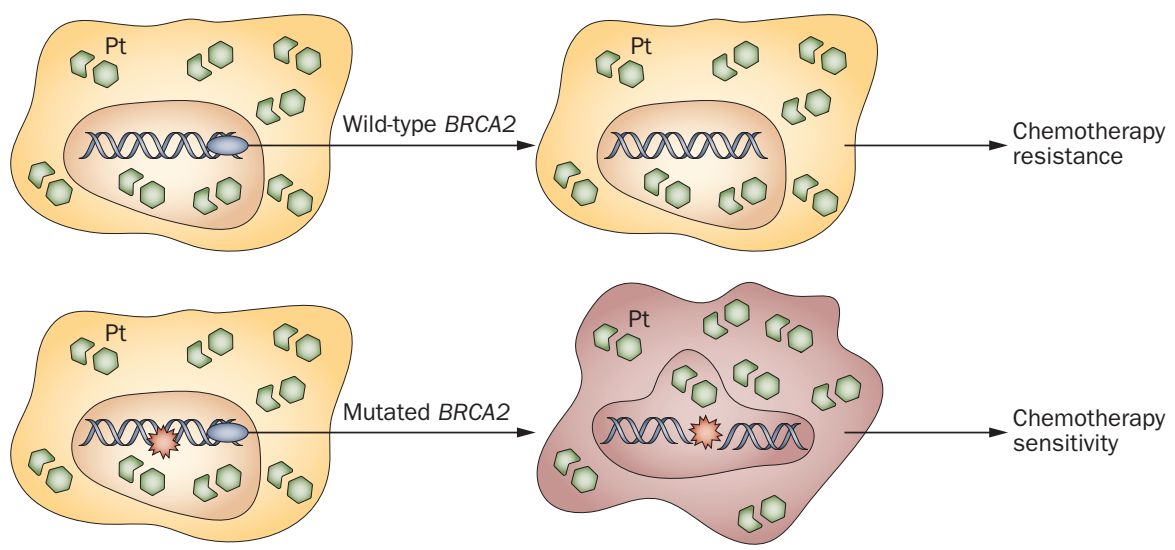

Original image courtesy of W. Zhang

that patients with mutations in BRCA2 but not in BRCA1 exhibited a 'mutator phenotype' that contained significantly more mutations than wild-type cases. Zhang elaborates, "the better responses of BRCA2-mutated cases are likely due to the highly mutated genome, or hypermutator phenotype, which not only provides tumors with a growth advantage but also exposes them to drug-induced DNA damage and cell death." Further work is needed to study BRCA1 and BRCA2, which seem to work differently in the DNA-repair process.

\section{Lisa Hutchinson}

Original article Yang, D. et al. Association of BRCA1 and BRCA2 mutations with survival, chemotherapy sensitivity, and gene mutator phenotype in patients with ovarian cancer. JAMA 306, 1557-1565 (2011) 